\title{
Aufsätze
}

Philip Manow und Peter Flemming

\section{Der Titel als politisches Distinktionsmerkmal?}

Eine Untersuchung akademischer und adeliger Wahlbewerber zum Deutschen Bundestag 1949-2009

\section{Kurzfassung}

Haben Wahlbewerber zum Deutschen Bundestag einen Wettbewerbsvorteil, wenn sie einen akademischen oder adeligen Titel tragen? In dieser Studie vergleichen wir Erststimmen- und Listenplatzunterschiede von Kandidaten mit und ohne Titel für alle Bundestagswahlen seit 1949. Die Untersuchung weist höhere Erststimmenanteile und bessere Listenplätze von Kandidaten mit Doktortitel oder adeligem Namenszusatz aus. Ob aber der Titel politischen Erfolg bewirkt, oder ob politisch erfolgreiche Bewerber mit einer höheren Wahrscheinlichkeit einen akademischen Titel erlangen, lässt sich auch auf Basis unserer umfangreichen Datengrundlage nicht endgültig entscheiden. 


\section{Inhalt}

1. Einleitung

2. Angebot und Nachfrage nach Akademikern und adeligen Kandidaten Wahlbewerber und Bundestagsmitglieder seit 1949

3. Determinanten des Wahlerfolgs: Macht der Titel einen Unterschied?

4. Fazit

\section{Einleitung}

Die zuletzt bekannt gewordenen Fälle von aufgrund von Plagiaten unrechtmäßig erworbenen Doktortiteln unter deutschen Politikern (zu Guttenberg, Koch-Mehrin, Chatzimarkakis) haben unter anderem zu der Frage geführt, ob der akademische Titel sich denn überhaupt in einen politischen Wettbewerbsvorteil übersetzt, und wenn ja, wie deutlich dieser Vorteil ausfällt. Ein möglicher Effekt ließe sich über den „Einfluss des Doktortitels auf den Wahlerfolg von Direktkandidaten“, das heißt über den Vergleich der Erststimmenanteile von Wahlbewerbern mit und ohne Titel ermitteln (Schneider/Tepe 2011). In der Studie von Schneider und Tepe kommen die Autoren anhand der Untersuchung der Erststimmenanteile der Direktkandidaten bei der Bundestagswahl von 2009 unter anderem zu dem Ergebnis, dass sich ein wenn auch insgesamt mäßiger - Effekt des Doktortitels auf den Erststimmenanteil nachweisen lässt, etwa in der Größenordnung eines Stimmenplus von 0,5 Prozentpunkten, wenn eine Reihe anderer Variablen kontrolliert wird, unter anderem die Anzahl aller Wahlbewerber mit Titel, die in einem Wahlkreis antreten. Dieser Befund deckt sich mit Untersuchungen, die zuvor bereits den Effekt bestimmter Kandidateneigenschaften, unter ihnen auch der akademische Titel, auf die Wahlergebnisse von Kandidaten oder ihrer Parteien zu ermitteln suchten (Rosar 2009; Kelley/ McAllister 1984; McDermott 1997, 2005; Mackenrodt 2008).

Im Folgenden beabsichtigen wir, über eine auf Direktkandidaten bzw. deren Stimmanteile beschränkte Betrachtung hinauszugehen und den Effekt akademischer Titel auf die Wahlchancen generell, das heißt auch für Listenkandidaten zu untersuchen. Hier stellt sich etwa die Frage, ob Bewerber mit einem akademischen Titel nicht auch auf besseren Listenplätzen platziert werden. Zugleich erweitern wir die Analyse um Namenszusätze, die auf adelige Familienabkunft hinweisen. Wir fragen nicht nur, ob der erworbene Doktortitel, sondern auch ob der ererbte Adelstitel einen messbaren Effekt auf die Erststimmen und auf die Listenplatzierungen hat. Zur Beantwortung unserer Fragen nutzen wir einen neuen Datensatz, der alle jemals in den 
bislang 17 Wahlen zum Deutschen Bundestag angetretenen Kandidaten und Kandidatinnen erfasst (Manow/Flemming 2011).

Wir geben zunächst einen kurzen Überblick über die Häufigkeit, mit der Promovierte und Adelige sich unter den Wahlbewerbern zum Deutschen Bundestag sowie unter den Bundestagsabgeordneten (MdBs) selbst befanden. Wir untersuchen den Erstimmeneffekt des akademischen Titels und des Adelstitels, deren Effekt auf die Listenplatzierungen und schließlich generell auf die Chancen der Wahlbewerber, in den Bundestag gewählt zu werden. Es zeigen sich zunächst für alle Dimensionen positive Effekte eines akademischen Titels auf den politischen Karriereverlauf, die nicht im gleichen Maß auch für Adelstitel bestätigt werden können. Zumindest der akademische Distinktionsgewinn scheint sich also in Vorteile im politischen Fortkommen zu übersetzen, auch wenn die ermittelten Effekte die konventionellen Grenzen statistischer Signifikanz oft (knapp) verpassen.

Unsere Analysen zeigen jedoch auch, dass sich grundsätzlich nicht ausschließen lässt, dass dies im Wesentlichen ein Effekt der Selbstselektion ist: Demnach würde der akademische Titel nur Persönlichkeitsmerkmale ausweisen, die besonders zur politischen Karriere befähigen, ohne dass das eine - der Titel - das andere - die Karriere - kausal beeinflussen würde. Einen solchen Effekt kann man auch für die ererbten Adelstitel nicht ausschließen, indizieren diese doch zumeist auch höhere Bildungsabschlüsse oder andere Persönlichkeitsaspekte, die auch der politischen Karriere förderlich sind. Methodisch kontrollieren lässt sich dieser Selbstselektionseffekt kaum - unser umfangreicher Datensatz erlaubt uns jedoch zumindest die Betrachtung von ca. 160 Kandidaten, deren Titelerwerb in den Beobachtungszeitraum fällt, so dass sich hier bei konstant gehaltenen Persönlichkeitsfaktoren ein nahezu ,reiner' Titeleffekt ermitteln lässt. Auch eine Fixed-Effects-Regression erlaubt es jedoch nicht, die Vermutung eines Selbstselektionseffekts zurückzuweisen. Wir berichten im Abschnitt 3 die entsprechenden Ergebnisse. Ein kurzer Ausblick schließt den Aufsatz ab.

\section{Angebot und Nachfrage nach Akademikern und adeligen Kandidaten - Wahlbewerber und Bundestagsmitglieder seit 1949}

Wir wollen zunächst fragen, wie sich in längerer Perspektive überhaupt das Angebot und die Nachfrage nach, ,akademischen Politikern“ bzw. nach solchen mit Adelstitel gestaltet. Heinrich Best und Maurizio Cotta hatten in ihren umfassend angelegten Studien zur parlamentarischen Repräsentation in Europa den Anteil von Akademikern und Adeligen unter den Parlamentariern als Indikator eines spezifischen Politiker- und Parteientypus - der Notablenpartei - interpretiert, der im Zuge der vollen 
Professionalisierung der politischen Klasse eher in Abnahme befindlich sei (Best/ Cotta 2000 a, 2000 b; Cotta/Best 2000). Insofern lohnt der über längere Zeiträume vergleichende Blick darauf, wie weitverbreitet generell zunächst akademische Titel unter den Wahlbewerbern wie auch sodann unter den gewählten Volksvertretern waren. Wir nutzen hierzu einen neuen Datensatz, der alle Kandidaten erfasst, die sich jemals bei einer Bundestagswahl um ein Mandat beworben haben. Der Datensatz umfasst 53.760 Kandidaturen (Personenjahre) und 35.211 verschiedene Kandidaten (Personen). Darunter sind 10.215 erfolgreiche Kandidaturen (Abgeordnetenpersonenjahre), die wiederum auf 3.581 verschiedenen Personen (Abgeordnete) beruhen. Der Datensatz enthält Informationen zur Kandidatur (Listen-, Wahlkreisoder Doppelkandidatur), zum Erststimmenergebnis, dem Listenplatz, der Parteizugehörigkeit, dem Geschlecht und Alter, zu Ausschussmitgliedschaften sowie eben auch Informationen zu (erworbenen) Doktoren- und Professorentiteln und (ererbten) Adelstiteln (Manow/Flemming 2011).

Schaubild 1: Häufigkeit akademischer Titel unter den Wahlbewerbern und den Mitgliedern des Bundestags (1949-2009)

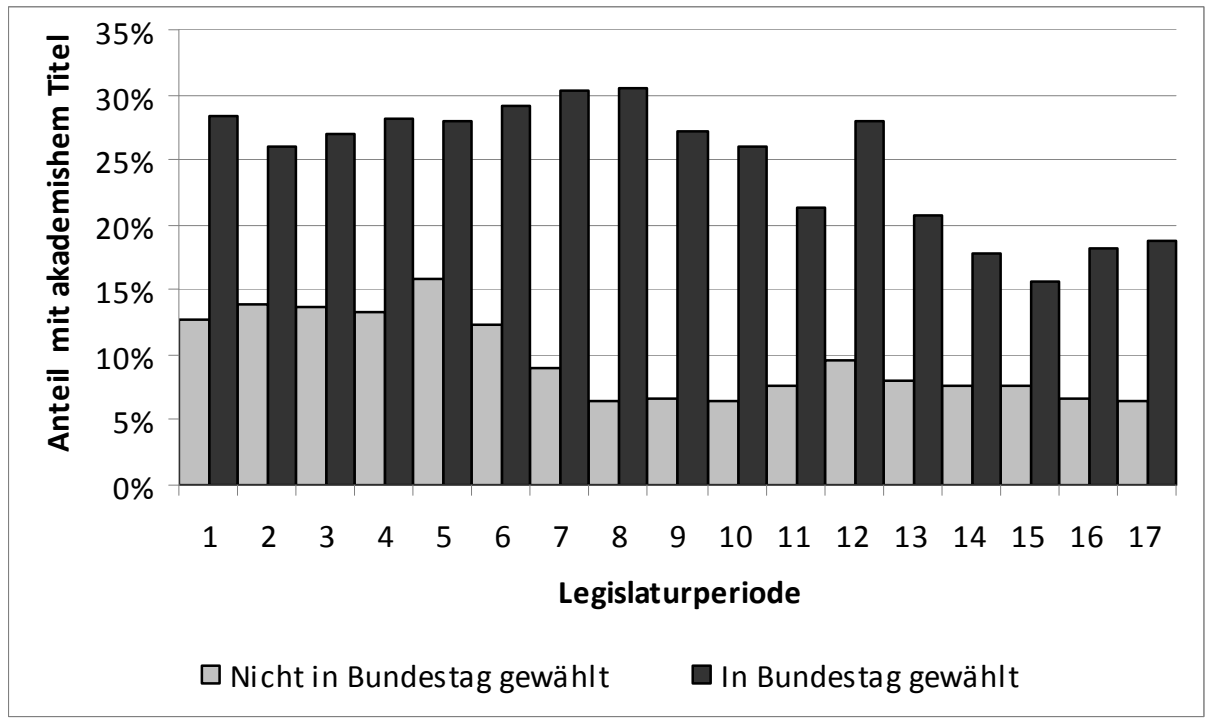

Anmerkung: Hervorzuheben ist der (leichte) Ausreißer nach oben in der 12. Legislaturperiode (1990-1994), in der 194 Doktoren und Professoren in Bundestag saßen, im Vergleich zu 146 in der Legislaturperiode zuvor. Diese erhöhte Zahl wurde im Wesentlichen durch Abgeordnete aus den neuen Bundesländern verursacht.

Quelle: Eigene Darstellung. 
Welches Bild ergibt nun eine erste Auswertung dieses Datensatzes im Hinblick auf Kandidaten und Abgeordnete mit akademischem Titel (Schaubild 1)?

Insgesamt zeigt sich im Zeitverlauf eine deutliche Abnahme der „Doktoren“ (und Professoren) unter Bewerbern wie Abgeordneten, im Gesamtkollektiv damit vermutlich die Zunahme des Distinktionsgewinns, der mit einem akademischen Titel für den Einzelnen verbunden ist. Unter den Bundestagsabgeordneten ist allerdings der akademische Titel mit nahezu 20\% auch heute noch recht weit verbreitet.

Im Falle zu Guttenbergs handelte es sich sogar um eine Kumulierung von Distinktionsmerkmalen: nicht nur die bürgerliche Auszeichnung, der juristische Doktortitel, sondern auch der ererbte Adelstitel. Was sind die politischen Effekte dieses Distinktionsmerkmals? Auch hier betrachten wir zunächst die Häufigkeit im Zeitverlauf, mit der sich Wahlbewerber mit adeligem Namenszusatz um ein Mandat im Bundestag beworben haben und wie oft sie dabei Erfolg hatten (Schaubild 2).

Schaubild 2: Wahlbewerber und Mitglieder des Bundestags mit Adelstitel (1949-2009)

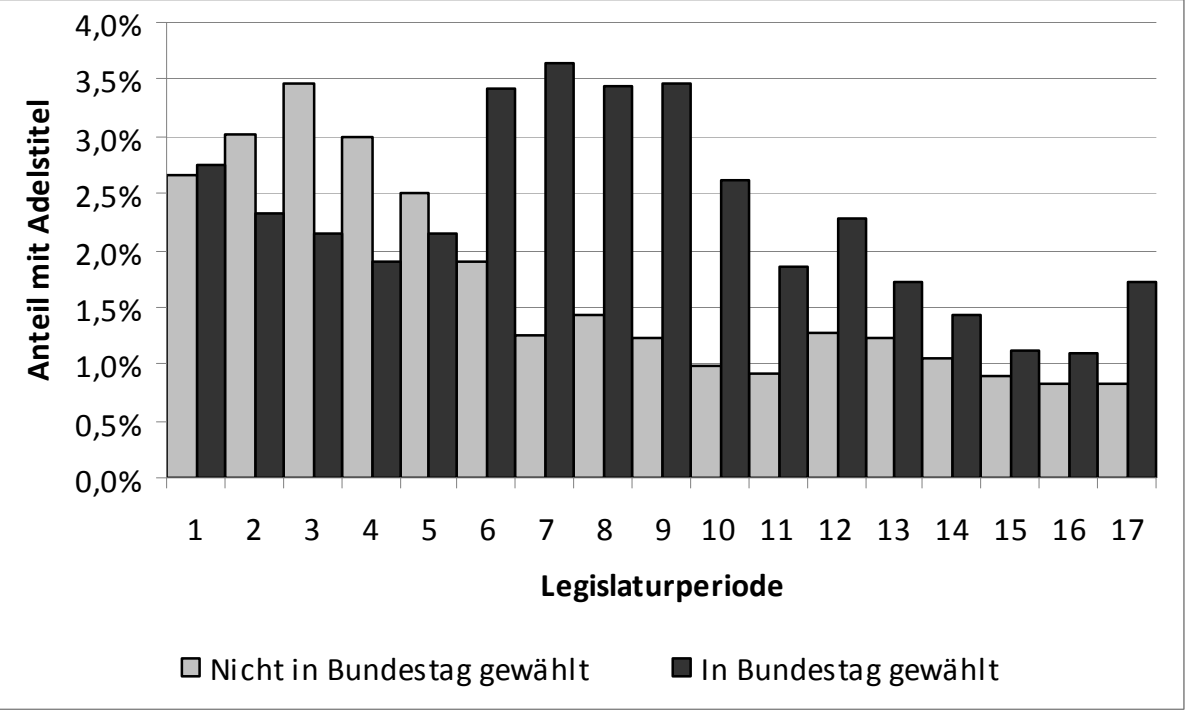

Quelle: Eigene Darstellung.

Es zeigen sich auch hier im Zeitverlauf deutlich zurückgehende Häufigkeiten, sowohl unter den Bewerbern als auch unter den gewählten Abgeordneten bei einem höheren Adelsanteil unter den MdBs - vor allem in den späteren Legislaturperioden. Insgesamt spielt sich diese zu den akademischen Titeln ähnliche Entwicklung aber 
auf einem deutlich niedrigeren Niveau ab: Der Vergleich zeigt zwischen 1,5\% und 3,5\% adelige Mitglieder des Bundestags und zwischen $12 \%$ bis $30 \%$ MdBs mit akademischem Titel.

Aufschlussreich ist allerdings die Aufgliederung der Titelhäufigkeiten nach Parteien, weil sich hier zeigt, dass Wahlbewerber mit Titel insbesondere im bürgerlichen Parteienspektrum häufiger zu finden sind. CDU/CSU und FDP weisen einen erheblich höheren Anteil von sowohl Promovierten als auch von Adeligen unter ihren Wahlbewerbern auf (siehe Schaubilder 3 a und 3 b). Für PDS/Die Linke ist auch hier der hohe Anteil der Doktoren unmittelbar nach der Wende hervorzuheben, ein Effekt, der sich jedoch auch für die SPD, CDU und FDP in der 12. Legislaturperiode zeigt: In Ostdeutschland hatten offensichtlich sowohl die Bürgerbewegung wie auch das regimetreue Milieu einen hohen Akademikeranteil.

Wenig überraschend ergeben sich auch bei den Adelstiteln sichtbar höhere Anteile im bürgerlichen Parteienspektrum, also bei CDU/CSU und FDP, wobei die CSU in den frühen Legislaturperioden hier die höchsten Werte - bis zu 8\% aller ihrer Wahlbewerber - aufweist.

Die ausgeprägten Unterschiede zwischen den Parteien werden erneut relevant, wenn wir die Listenplatzierungen der Wahlbewerber mit akademischem Titel betrachten. Dies ist Gegenstand des nachfolgenden Abschnitts. 
Der Titel als politisches Distinktionsmerkmal?

Schaubild 3 a: Wahlbewerber mit akademischem Titel (1949-2009, nach Parteien)

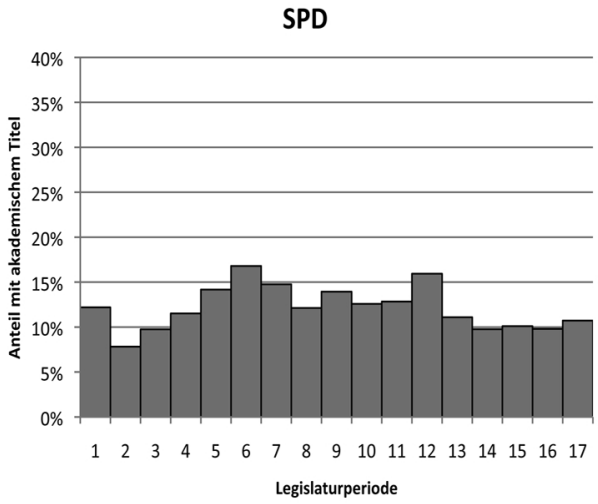

CSU

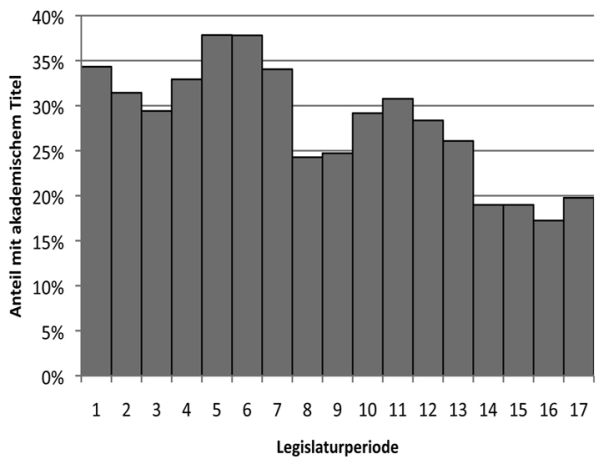

B90/Grüne

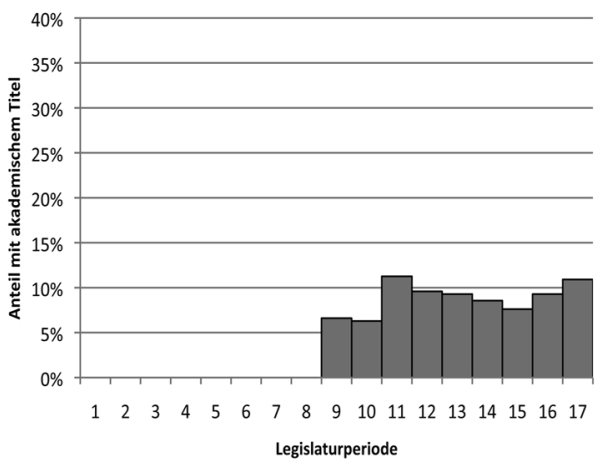

Quelle: Eigene Darstellung.
CDU

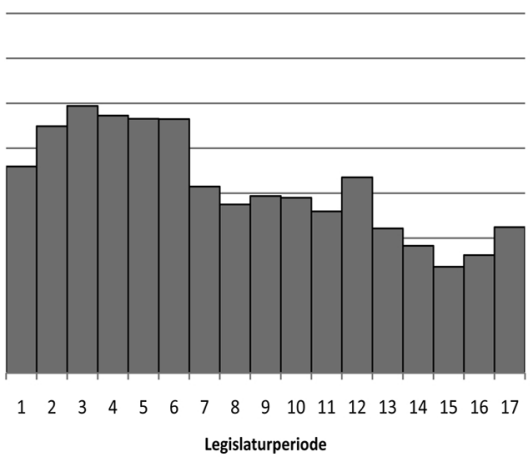

FDP

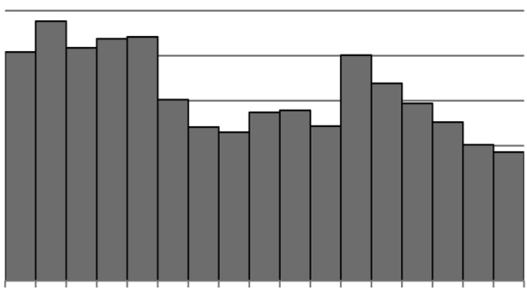

$\begin{array}{lllllllllllllllll}1 & 2 & 3 & 4 & 5 & 6 & 7 & 8 & 9 & 10 & 11 & 12 & 13 & 14 & 15 & 16 & 17\end{array}$ Legislaturperiode

\section{Linke/PDS}

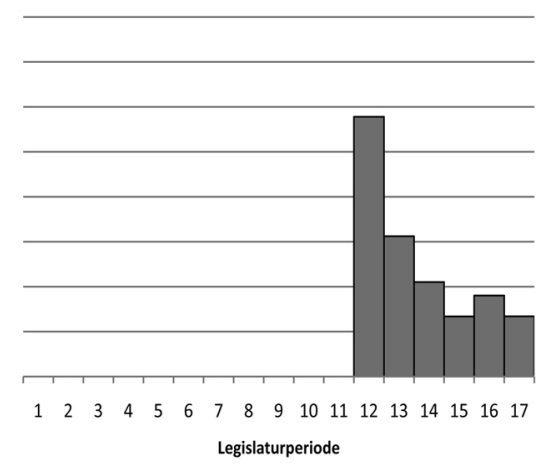


Schaubild 3 b: Wahlbewerber mit Adelstitel (1949-2009, nach Parteien)

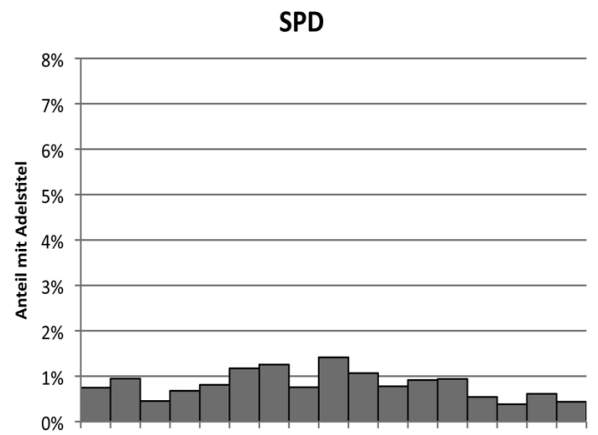

$\begin{array}{lllllllllllllllll}1 & 2 & 3 & 4 & 5 & 6 & 7 & 8 & 9 & 10 & 11 & 12 & 13 & 14 & 15 & 16 & 17\end{array}$ Legislaturperiode

CSU

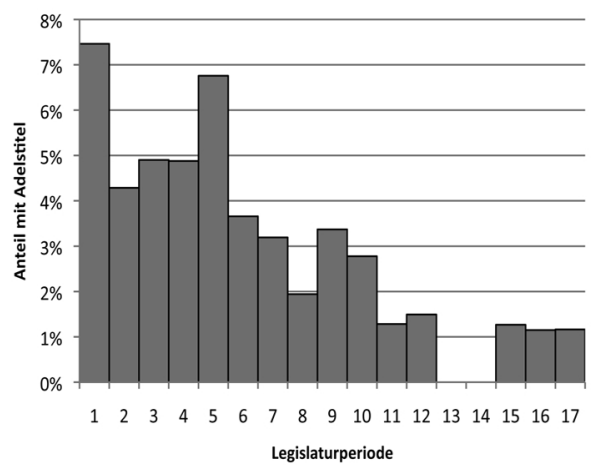

B90/Grüne

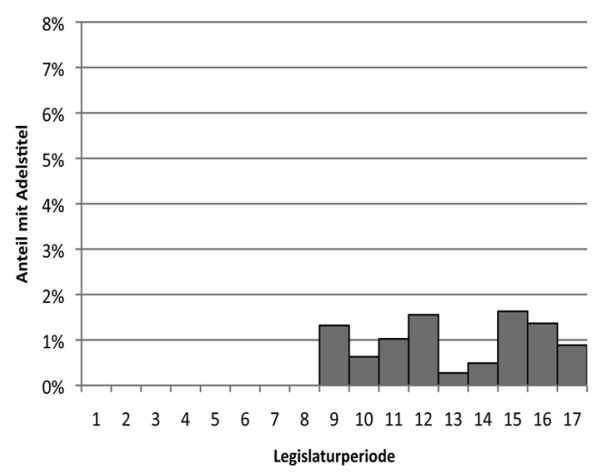

CDU

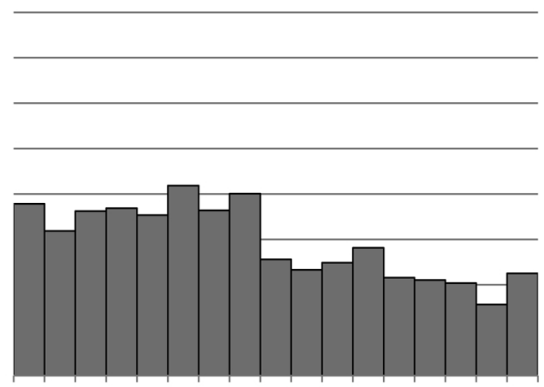

$\begin{array}{lllllllllllllllll}1 & 2 & 3 & 4 & 5 & 6 & 7 & 8 & 9 & 10 & 11 & 12 & 13 & 14 & 15 & 16 & 17\end{array}$ Legislaturperiode

FDP

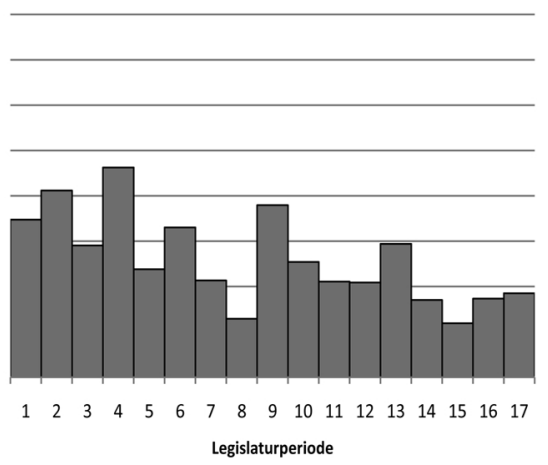

Linke/PDS

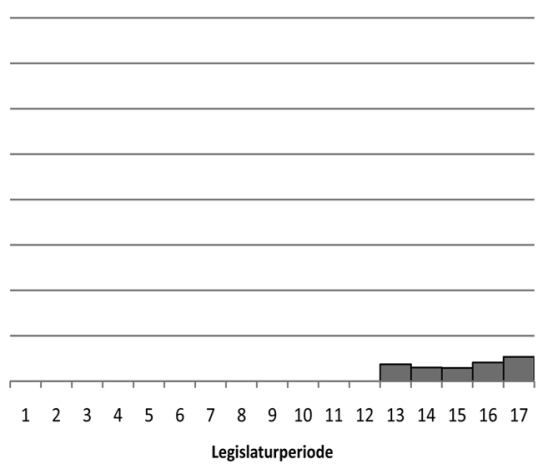

Quelle: Eigene Darstellung. 


\section{Determinanten des Wahlerfolgs: Macht der Titel einen Unterschied?}

Wenn wir für den gesamten Zeitraum die grundsätzliche Frage nach dem politischen Erfolgswert eines akademischen Titels stellen, bestätigt sich zunächst der in vorherigen Studien gefundene positive Effekt des akademischen Titels auf das Erststimmenergebnis, und zwar in etwa der Größenordnung, die auch in der Literatur berichtet wird, allerdings nur an der Grenze zur Signifikanz (siehe Tabelle 1; Modell 1). Das Stimmenplus des Titels beträgt etwa $0,7 \%$ - kein sehr starker, doch auch ein im Kandidatenwettkampf nicht zu vernachlässigender Effekt. Hinzuweisen ist allerdings darauf, dass sich dieser Effekt in unserem Datensatz im Vergleich zu allen im Wahlkreis erfolgreichen Bewerbern, also zu allen anderen Wahlkreisabgeordneten, ergibt, nicht - wie in anderen Studien - im Vergleich aller Wahlkreisbewerber. Damit ist die Vergleichsgruppe nun aus den anderen erfolgreichen Wahlbewerbern gebildet, die Hürden für das Auftreten eines signifikanten Erstimmenvorteils durch den akademischen Titel sind also höher gelegt.

Tabelle 1: Erststimmenergebnis bei Direktwahl

\begin{tabular}{|l|c|c|}
\hline & Modell 1 & Modell 2 \\
\hline Doktortitel & 0,716 & 0,710 \\
\hline & $(0,146)$ & $(0,144)$ \\
\hline Adelstitel & & 1,335 \\
\hline & & $(0,400)$ \\
\hline Konstante & 49,661 & 49,637 \\
\hline & $(0,000)^{* * *}$ & $(0,000)^{* * *}$ \\
\hline Beobachtungen & 4.605 & 4.605 \\
\hline Adjusted R-squared & 0,001 & 0,001 \\
\hline
\end{tabular}

Anmerkung: Robuste p-Werte in Klammern; * signifikant auf dem 10\%-Niveau; ** signifikant auf dem 5\%-Niveau; ${ }^{* * *}$ signifikant auf dem $1 \%$-Niveau.

Quelle: Eigene Darstellung.

Die Frage nach dem Erststimmeneffekt sowohl des erworbenen wie des ererbten Titels beantwortet Modell 2 aus Tabelle 1. Die Regressionskoeffizienten weisen einen etwa doppelt so großen Effekt des Adelstitels aus - ein durchschnittliches Stimmenplus von 1,3\%-, aber dieser Koeffizient verfehlt konventionelle Standards statistischer Signifikanz deutlich.

Unsere bisherige Betrachtung war - wie auch in der Mehrzahl der Studien zum politischen Titeleffekt - auf die Direktkandidaten beschränkt. Bei der Antwort auf 
die Frage nach dem politischen Erfolgswert des Doktor- wie des Adelstitels ist es aber womöglich aus Sicht der Kandidaten (und daher auch für uns) von Interesse, ob der Titel nicht auch für die Listenpositionierung einen Unterschied macht, insbesondere, ob Parteien die Kandidaten mit Titel nicht auch besser platzieren.

Es scheint plausibel, dass nicht nur die Kandidaten ein Interesse an der politischen Nutzung ihres Titels haben, sondern Parteien ebenso auf einen Reputationsgewinn spekulieren, wenn sie unter ihren Listenkandidaten mit höheren Erfolgschancen also besseren Listenpositionen - Bewerber mit akademischen oder adeligen Titeln führen. In diesem Zusammenhang bekommt ein technisches Merkmal, nämlich die Namensnennung auf dem Stimmzettel, Bedeutung. Auf den Bundestagswahlzetteln werden laut Bundeswahlordnung für die Zweitstimme die ersten fünf Positionen der betreffenden Landesliste namentlich aufgeführt (siehe Bundeswahlordnung $\S 45$ Abs. 1 Satz 2). Zudem ist der Titel - ob akademisch oder adelig - als gesetzlicher Namenszusatz anzugeben. ${ }^{1}$ Sollte sich also der Wähler von der Information, die ein Titel vor dem Namen auf dem Wahlzettel darstellt, beeinflussen lassen, wie in der Literatur angenommen und bisweilen bestätigt wurde (McDermott 2005; Kelley/ McAllister 1984; Rosar 2009; Schneider/Tepe 2011; Mackenrodt 2008), so wäre schwer zu begründen, warum sich dieser Titeleffekt auf die Erststimme und mithin die Direktkandidaten beschränken sollte. Anders formuliert: Ein Titel könnte nicht nur ein zwischenparteilicher, sondern auch ein innerparteilicher Wettbewerbsvorteil sein, wenn Parteien in Antizipation eines Titeleffekts auf die Wahlentscheidung der Stimmbürger die Neigung haben, Titelträger überdurchschnittlich häufig auf ihre ersten fünf Positionen auf der Landesliste zu platzieren.

1 Bundeswahlordnung in der Fassung der Bekanntmachung vom 19. April 2002 (BGB1. I S. 1376), zuletzt geändert durch Art. 1 der Zweiten Verordnung zur Änderung der Bundeswahlordnung und der Europawahlordnung vom 3. Dezember 2008 (BGBl. I S. 2378). Hier heißt es in $\S 45$ Abs. 1: „Der Stimmzettel enthält nach dem Muster der Anlage 26 je in der Reihenfolge und unter der Nummer ihrer Bekanntmachung

1. für die Wahl im Wahlkreis in schwarzem Druck die zugelassenen Kreiswahlvorschläge unter Angabe des Familiennamens, Vornamens, Berufs oder Standes und der Anschrift (Hauptwohnung) des Bewerbers sowie des Namens der Partei, sofern sie eine Kurzbezeichnung verwendet, auch dieser, $[\ldots .$.

2. für die Wahl nach Landeslisten in blauem Druck die zugelassenen Landeslisten unter Angabe des Namens der Partei, sofern sie eine Kurzbezeichnung verwendet, auch dieser, sowie der Familiennamen und Vornamen der ersten fünf Bewerber und links von der Parteibezeichnung einen Kreis für die Kennzeichnung." Der zweimalige Hinweis auf den Familiennamen ist wichtig, weil sowohl der akademische Titel als auch der Adelstitel gesetzliche Namenszusätze sind. Dies bedeutet beispielsweise, dass auch Kandidaten wie Dr. Hermann Otto Solms oder Jutta Ditfurth, die konsequent auf das Führen ihres Adelstitels verzichten, auf den Wahlzetteln unter voller Titelnennung, also als Prinz zu Solms Hohensolms-Lich bzw. als Jutta von Dittfurth erscheinen. 
Zur Beantwortung der Frage nach dem Listenplatzeffekt ermitteln wir zunächst eine relative Listenposition, das heißt wir teilen den tatsächlichen Listenplatz des einzelnen Bewerbers durch die Gesamtlänge der Liste. Die relative Position reicht dann von $\approx 0$ bis 1 . Wir kontrollieren hiermit die unterschiedliche Länge der Parteilandeslisten ${ }^{2}$ und betrachten zunächst, ob ein Doktor- oder ein Adelstitel auch mit einer besseren (relativen) Listenpositionierung korreliert (Tabelle 2)

Tabelle 2: Korrelation von akademischen und Adelstitel mit relativer Listenposition

\begin{tabular}{|l|c|c|c|}
\hline & Relativer Listenplatz & Akademischer Titel & Adelstitel \\
\hline Relativer Listenplatz & 1,0000 & & \\
\hline Akademischer Titel & $-0,1503$ & 1,0000 & \\
\hline Adelstitel & $-0,0191$ & 0,0422 & 1,0000 \\
\hline
\end{tabular}

Quelle: Eigene Darstellung.

Wie ersichtlich ist, besetzen promovierte, kaum aber adelige Wahlbewerber tatsächlich bessere Listenplätze. Sie sind deutlich besser platziert, nämlich um ca. 15\%. Um die Effektgröße abzuschätzen: Im Durchschnitt besaßen alle Landeslisten in allen Bundestagswahlen seit 1949 eine Länge von 20 Plätzen - der Träger eines Doktortitels wäre auf einer Landesliste dieser Länge durchschnittlich auf einer um 3 Listenplätze besseren Position zu finden als der Kandidat ohne Titel. Dies ist ein durchaus substanzieller Effekt. Um es jedoch nochmals zu betonen: In welchem Ausmaß dies durch den Doktortitel verursacht ist, oder den sonstigen einer politischen Karriere förderlichen Fähigkeiten, Charaktereigenschaften und Persönlichkeitsmerkmalen des jeweiligen Wahlbewerbers geschuldet ist, die der Doktortitel lediglich indiziert, muss offen bleiben. ${ }^{3}$ Zumindest gäbe es auch unter der Annahme eines Selbstselektionseffekts aus Sicht einzelner Wahlbewerber einen Anreiz zum Titelerwerb aus Mimikry, wenn der Titel - von Wählern wie Parteien - als ein Näherungswert für andere wünschbare Kandidatenmerkmale interpretiert wird. Für

2 Natürlich sollte ein solcher Listeneffekt des Titels nicht für alle Landeslisten relevant sein - bekanntlich gibt es Landeslisten, über die kaum ein oder kein einziger Kandidat in den Bundestag einzieht. Doch bei der großen Fallzahl in unserem Datensatz sollte sich ein Durchschnittseffekt auch dann zeigen, wenn wir nicht zwischen aussichtsreichen und aussichtslosen Landeslisten unterscheiden.

3 Unser Datensatz enthält 163 Personen, die ihren Doktortitel erst im Beobachtungszeitraum erworben haben, von diesen sind 156 Beobachtungen für die Betrachtung der Listenposition relevant. Der Vergleich des Listenplatzes oder des Erststimmenergebnisses identischer Personen mit und ohne Titel in einem Fixed-Effects-Modell erlaubt die Ermittlung eines Titeleffekts bei weitgehender Kontrolle weiterer persönlicher Merkmale (Brüderl 2010); siehe auch die Ergebnisse in Tabelle 4. 
die adeligen Wahlbewerber ist der Effekt hingegen gering - mit einem Adelstitel könnte man sich nur um weniger als einen halben Listenplatz verbessern.

Wie bereits erläutert, sollten - wenn Titelträger mit einem Reputations- und damit Stimmengewinn bei den Wählern rechnen können - nicht nur die einzelnen Wahlbewerber, sondern auch die sie aufstellenden Parteien hieraus politisches Kapital zu schlagen versuchen. Wenn die Zusatzinformation, die in einem gesetzlichen Namenszusatz wie dem akademischen Titel enthalten ist, für eine gewisse Zahl von Wählern politisch informationsträchtig, also ihre Wahlentscheidung zu beeinflussen in der Lage ist, sollten dies nicht nur Direktkandidaten im Wahlkreis, sondern auch die Parteien bei der Listenaufstellung in Rechnung stellen. Mittlerweile ist bekannt, dass die Reihenfolge der Namensnennung auf den Parteilisten einen Effekt auf die Erststimmenergebnisse der jeweiligen Kandidaten hat (Faas/Schoen 2006). Thorsten Faas und Harald Schoen vermuten, dass Variablen, die auf die eine oder andere Art die Prominenz eines Wahlbewerbers indizieren, und hierunter fassen sie insbesondere auch akademische Titel, ,are likely to be correlated positively with both ballot position and relative vote shares” (ebd.: 96). Die „ballot position“ gewinnt insbesondere dann an Relevanz, wenn die ersten Listenpositionen - wie auf den Stimmzetteln für die Bundestagswahlen - auf dem Wahlzettel namentlich aufgeführt werden. Dies führt zu der Vermutung, dass sich ein akademischer Titel - wenn er denn ein politisch vorteilhaftes Distinktionsmerkmal ist - gehäuft unter den oberen Listenpositionen finden sollte. Dies wiederum würde die Wahlchancen der Titelträger unter den Wahlbewerbern erhöhen. ${ }^{4}$

Und in der Tat, die Häufigkeitsverteilung des akademischen Titels differenziert nach Listenpositionen weist nicht nur einen klar abnehmenden Trend auf (siehe Schaubild 4), sondern zugleich auch offensichtlich zwei Sprünge. Einmal ist die Häufigkeit von akademischen Titeln auf dem prominentesten, herausgehobenen Listenplatz 1 bemerkenswert. Sie erklärt mit Sicherheit auch einen Teil des Erststimmeneffekts akademischer Titel, denn auf dem ersten Platz der Landeslisten findet sich die jeweilige Parteiprominenz, die auch im Wahlkreis im Regelfall überdurchschnittliche Ergebnisse realisiert. Ein zweiter, kleinerer Sprung wird zwischen Platz 5 und 6 sichtbar, genau dann, wenn der Titel als Namenszusatz zum letzten Mal eine Zusatzinformation auf dem Stimmzettel bieten kann. Während die abnehmende Häufigkeit von akademischen Titeln mit höheren Listenplätzen wiederum

4 Natürlich bieten Landeslisten je nach Parteien ganz unterschiedliche politische Karriereaussichten. So ziehen Kandidaten von bestimmten Parteien in bestimmten Bundesländern fast ausschließlich direkt über den Wahlkreis in den Bundestag ein, etwa Kandidaten der CSU in Bayern oder der SPD in Brandenburg oder Bremen. Angesichts der hohen Fallzahl unseres Datensatzes sollte sich dennoch im Durchschnitt ein Listeneffekt zeigen, wenn er denn vorhanden ist. 
auch ein reiner Selbstselektionseffekt sein kann - politischer wie akademischer Erfolg verweisen beide auf dahinterliegende Erfolgsmerkmale der Person -, so wäre eine überzufällige Abnahme der Titelhäufigkeiten zwischen Rang 5 und 6 schwerer mit einer solchen Erklärung und leichter mit einer Erklärung zu vereinbaren, die auf solche Heuristiken verweist, aufgrund derer Wähler mit schwacher Parteipräferenz ihre Wahlentscheidung treffen (Kelley/McAllister 1984; McDermott 2005, 1997) und auf die Art und Weise, wie Parteien das Wissen um diese Entscheidungsheuristiken strategisch nutzen.

Schaubild 4: Häufigkeit von akademischen Titeln nach Listenplätzen (alle Wahlbewerber 1949-2009)

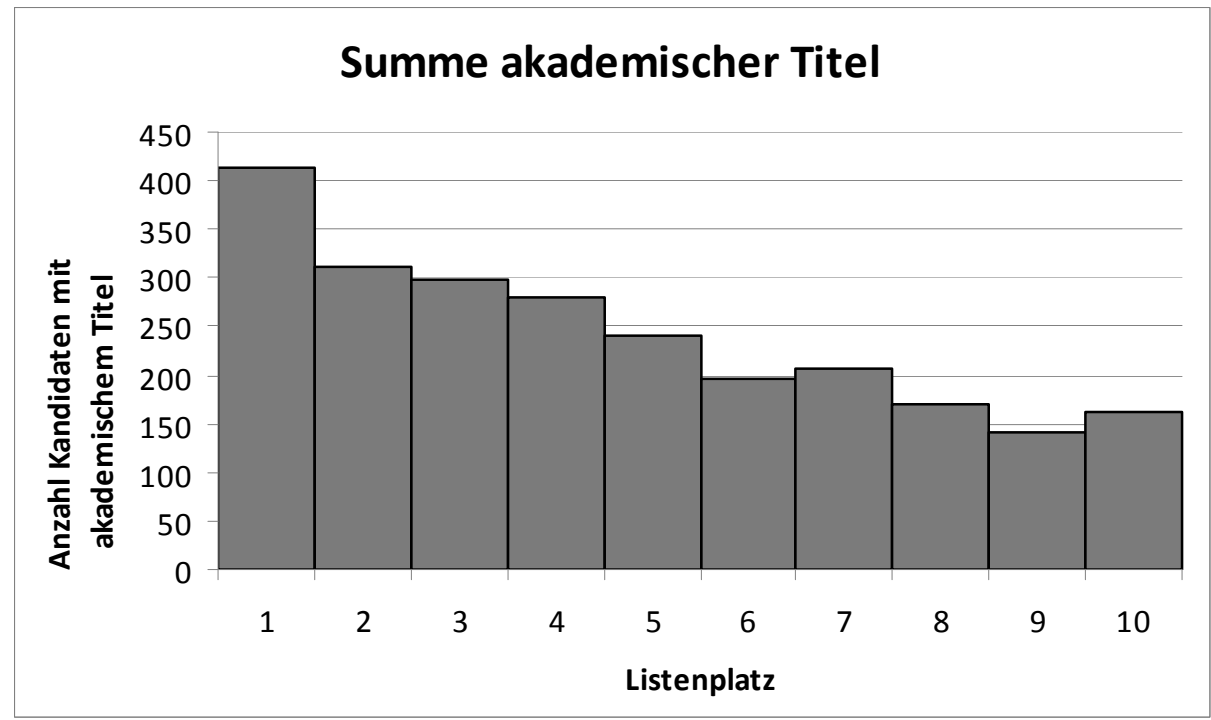

Quelle: Eigene Darstellung.

Das führt zu der Frage, a) ob es denn einen solchen überzufälligen Sprung tatsächlich gibt, und b) ob sich hier Parteiunterschiede zeigen?

Zunächst ergibt der Mittelwertvergleich über einen t-Test, dass die unterschiedlichen Häufigkeiten, mit denen Titelträger entweder auf Platz 5 oder Platz 6 einer Landesliste $\mathrm{zu}$ finden sind, nur auf einem Niveau von 10-\% statistisch signifikant sind (mit einem t-Wert von 1,32 und einer Wahrscheinlichkeit $\operatorname{Pr}(\mathrm{T}>\mathrm{t})=0,0938$ ). Schränken wir die Betrachtung auf die etablierten, das heißt auf die im Bundestag vertretenen Parteien ein, verschwindet der Zusammenhang. Schließlich zeigt auch die nach Parteien differenzierte Betrachtung, dass dieser Rangeffekt offensichtlich 
ein reines Phänomen der sonstigen Parteien ist (siehe Schaubild 5). Sind diese ParteiAußenseiter besonders darauf angewiesen, dem Wähler über Kandidaten mit akademischem Titel Seriosität zu signalisieren? Für die CDU und die FDP, bei denen die Aufstellung akademisch ausgewiesener Kandidaten am häufigsten vorkommt und vermutlich am ehesten auf die Reflexe einer bürgerlichen Klientel zu zielen vermag, zeigt sich der Sprung zwischen Rangplatz 5 und 6 allerdings nicht (auf niedrigem Niveau jedoch offensichtlich für die CSU).

Ohne die sonstigen Parteien nimmt der Anteil der Titelträger mit steigendem Listenplatz eher linear ab - ein Befund, der wiederum eher mit der Selbstselektionshypothese vereinbar ist. Sie scheint zumindest für die etablierten Parteien Geltung zu besitzen. Die namentliche Erwähnung auf dem Stimmzettel, entweder an herausgehobener erster Position oder überhaupt, verleitet also offensichtlich nur Außenseiter-Parteien dazu, häufiger promovierte Wahlbewerber in die Gruppe der fünf Namen aufzunehmen, die für die Zweitstimme aufgelistet werden (siehe Bundeswahlordnung § 45 Abs. 1 Satz 2). Anders formuliert: diese weisen promovierten Wahlbewerbern häufiger die vorderen fünf Listenplätze zu. Hier geht es vermutlich um das Aussenden von Seriositätssignalen an Wähler, die über die betreffenden Parteien nur wenige Informationen besitzen. Lässt sich ein ähnlicher Befund auch für die adeligen Wahlbewerber treffen?

Wenn wir analog nach den Listenplatzierungen der Wahlbewerber mit Adelstitel fragen, so ergibt sich nach Parteien differenziert das in Schaubild 6 dargestellte Bild.

Erneut sind es - nicht wirklich überraschend - die bürgerlichen Parteien, bei denen sich die Wahlbewerber mit Adelstitel häufiger finden. Sowohl für die CDU als auch für die FDP zeigt sich hier offensichtlich ein Sprung vom fünften zum sechsten Listenplatz, während nun bei den Sonstigen ein genau entgegengesetztes Muster erkennbar wird, nämlich eine höhere Anzahl adeliger Wahlbewerber auf dem ,unsichtbaren“ sechsten als auf dem ,sichtbaren“ fünften Listenplatz. Ein Mittelwertevergleich für die etablierten Parteien (von der SPD bis einschließlich PDS/ Die Linke) weist auch tatsächlich die unterschiedlichen Häufigkeiten als überzufällig aus (mit einem $t$-Wert von 1,479, $\operatorname{Pr}(T>t)=0,0696$ ). Diese Befunde basieren jedoch insgesamt auf einer eher kleinen Fallzahl. 
Schaubild 5: Akademische Titel nach Partei und Rangplatz
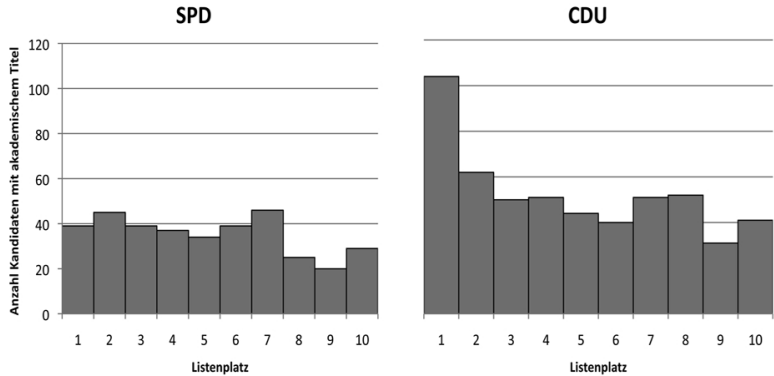

FDP

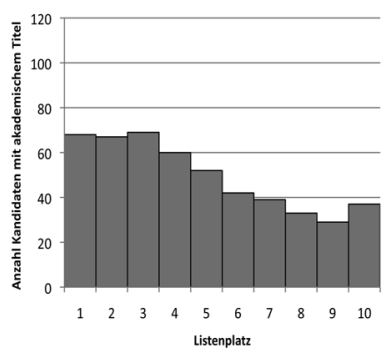

B90/Grüne

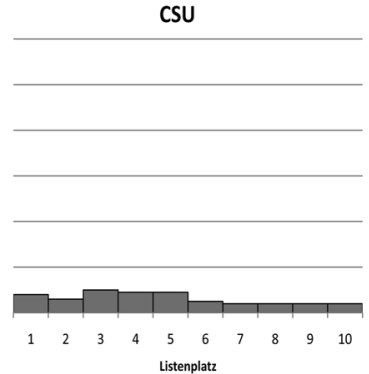

NPD
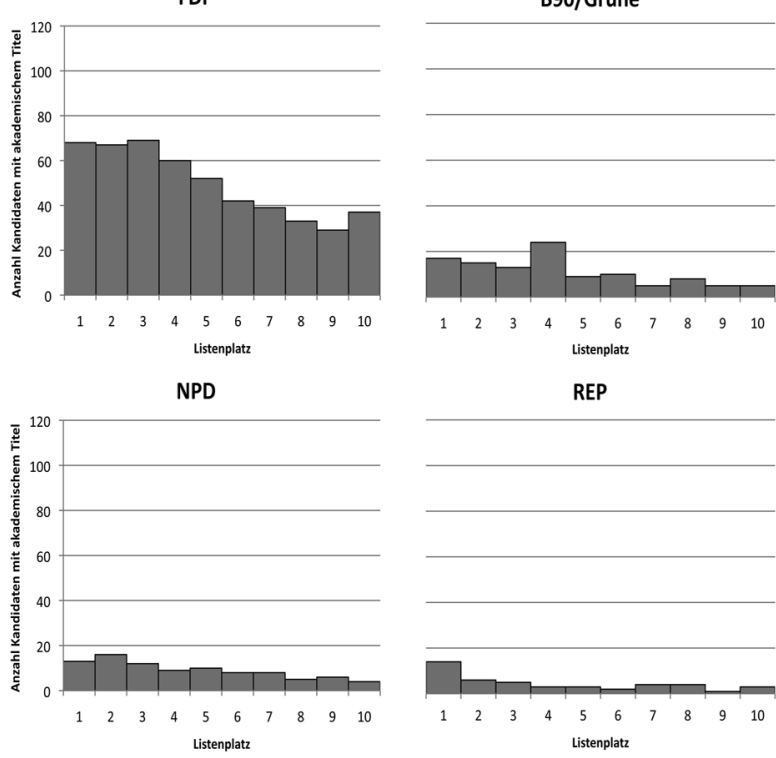

istenplat

Linke/PDS
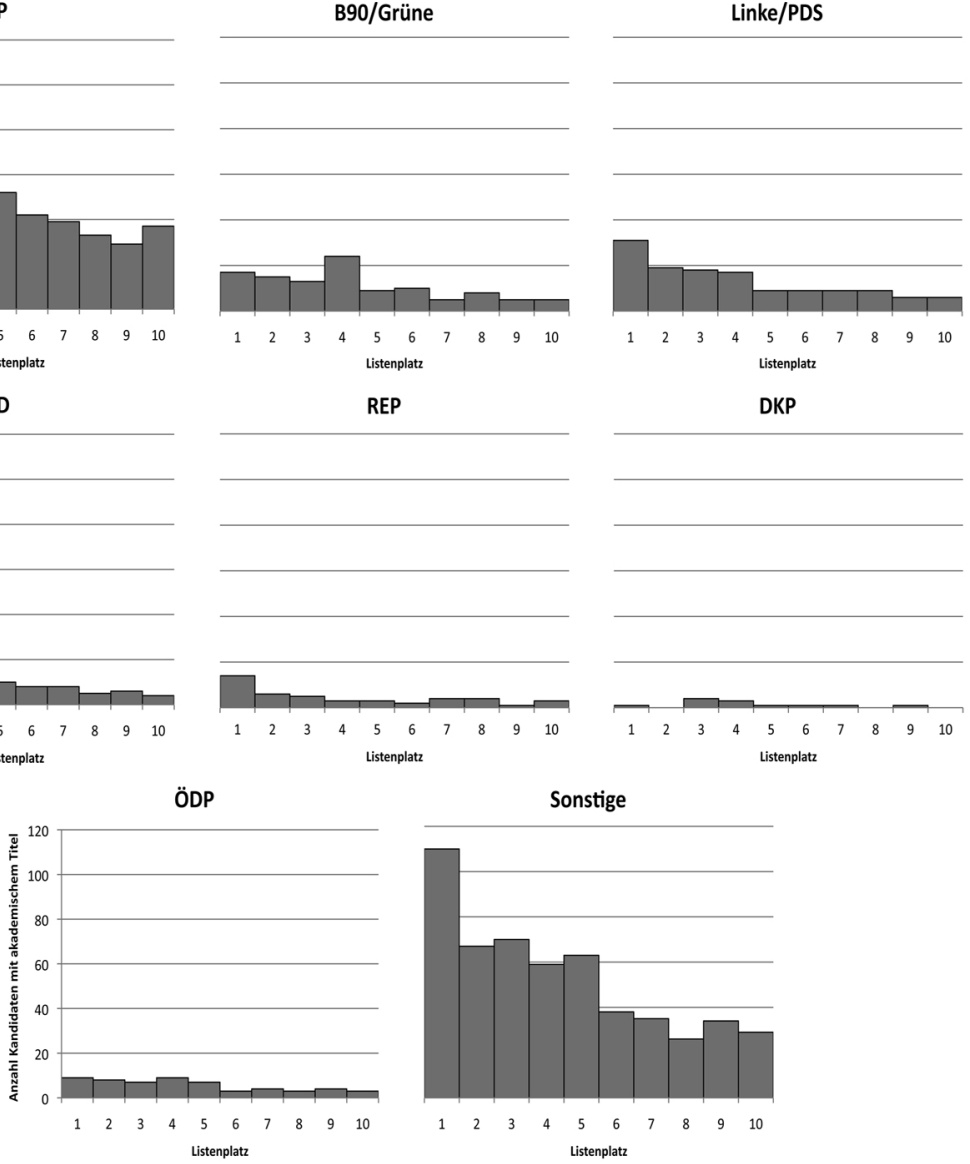

Quelle: Eigene Darstellung. 
Schaubild 6: Adelstitel nach Partei und Rangplatz
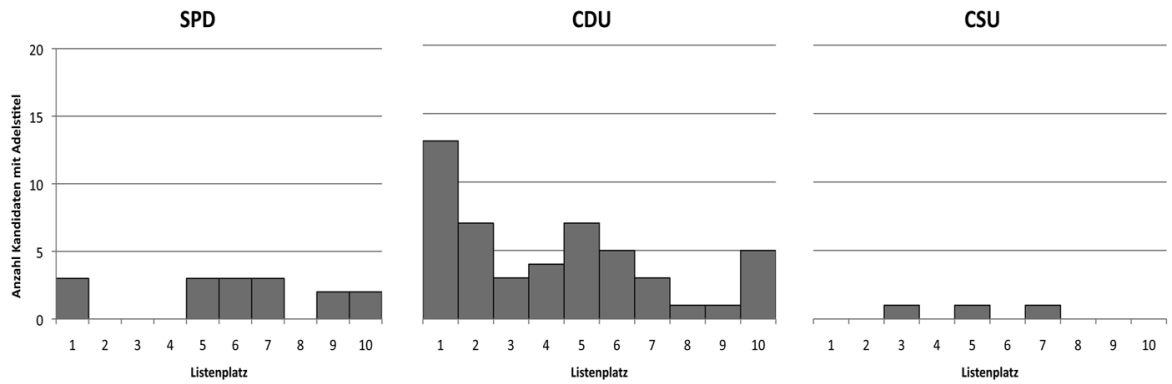

FDP

B90/Grüne
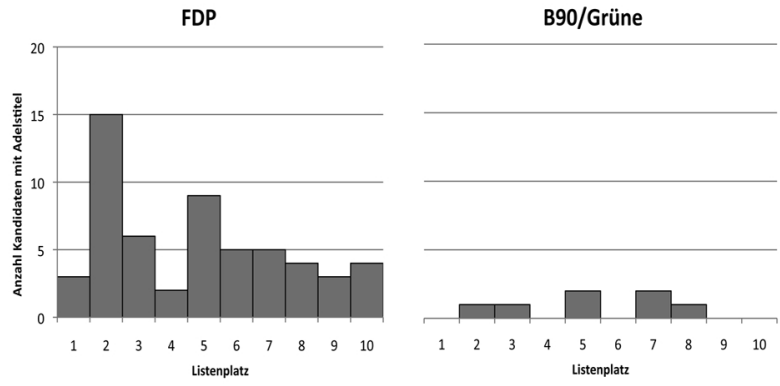

Linke/PDS
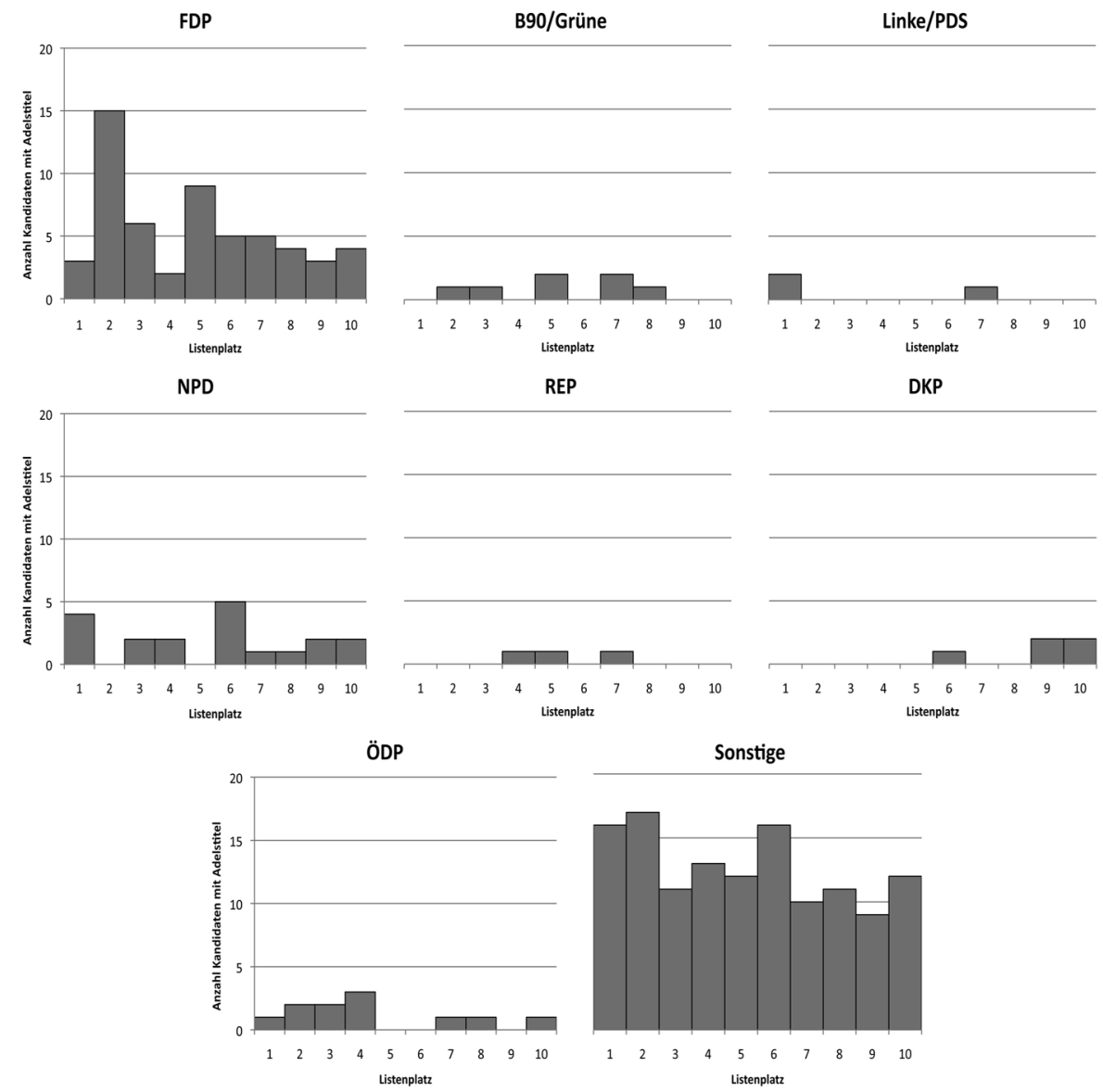

Quelle: Eigene Darstellung. 
Wir kommen nun abschließend zur Überprüfung der Frage, inwieweit generell der Titel die Chancen von Kandidaten erhöht, gewählt zu werden. Wir untersuchen mit einer logistischen Regression die Chance, in den Bundestag gewählt zu werden in Abhängigkeit vom Tragen eines Titels (Tabelle 3). Zunächst betrachten wir alle Kandidaten, anschließend - um den Erststimmeneffekt vom Listeneffekt zu trennen - die Chancen für die nicht über die Liste bzw. nicht durch den Wahlkreis in den Bundestag gekommenen Abgeordneten.

Tabelle 3: Logistische Regression, Determinanten des Wahlerfolgs, Odds Ratios

\begin{tabular}{|l|c|c|c|}
\hline & $\begin{array}{c}\text { Alle } \\
\text { (A) }\end{array}$ & $\begin{array}{c}\text { Direkt } \\
\text { (B) }\end{array}$ & $\begin{array}{c}\text { Liste } \\
\text { (C) }\end{array}$ \\
\hline Adelstitel & 1,281 & 1,163 & 1,385 \\
\hline & $(0,070)^{*}$ & $(0,411)$ & $(0,047)^{* *}$ \\
\hline Doktortitel & 3,156 & 3,358 & 2,783 \\
\hline & $(0,000)^{* * *}$ & $(0,000)^{* * *}$ & $(0,000)^{* * *}$ \\
\hline Beobachtungen & 53.760 & 48.919 & 49.169 \\
\hline
\end{tabular}

Anmerkungen wie Tabelle 1.

Quelle: Eigene Darstellung.

Tabelle 3 weist Odds Ratios aus, die direkt zu interpretieren sind. Vier Ergebnisse verdienen dabei festgehalten zu werden: (1) Zunächst gehen beide Titelformen mit einer sichtbar höheren Wahrscheinlichkeit, in den Bundestag gewählt zu werden, einher (Spalte A). Hierbei fällt (2) der akademische Titeleffekt sehr viel deutlicher aus als der adelige: Der promovierte Wahlbewerber hat eine 3,2-fach erhöhte Wahlwahrscheinlichkeit als der nicht-promovierte, der adelige eine 1,3-fach erhöhte Wahlwahrscheinlichkeit als der nicht-adelige. (3) Wenn der Adelstitel überhaupt einen Effekt hat, dann offensichtlich durch eine bessere Listenpositionierung adeliger Wahlbewerber (Spalte C), während (4) der Effekt des akademischen Titels stärker im Wahlkreis als bei der Listenerstellung zum Tragen kommt (Spalte B), obwohl auch ein deutlicher Listeneffekt ersichtlich wird.

Alle unsere bisherigen Analysen erlauben es jedoch weiterhin nicht, einen reinen Titeleffekt von einem Effekt der Selbstselektion zu trennen: Erklärt ein Titel (zumindest partiell) den politischen Erfolg, oder haben erfolgreiche Politiker nur häufiger einen Titel? Die Größe unseres Kandidatendatensatzes ermöglicht jedoch die Betrachtung derjenigen Kandidaten (156), bei denen der Zeitpunkt des Erwerbs 
eines akademischen Titels innerhalb der Betrachtungsperiode liegt. ${ }^{5}$ Mit dem Vergleich der Episoden vor und nach dem Titelerwerb können wir die jeweiligen Persönlichkeitsmerkmale weitgehend konstant halten. In einem sehr einfachen FixedEffects-Modell, das zunächst nur zusätzlich für das Alter kontrolliert, zeigt sich der erwartete positive Effekt des Titels auf die Listenplatzierung - wenngleich wiederum konventionelle Standards statistischer Signifikanz verfehlt werden und die Effektstärke gering bleibt. Gleiches gilt für den Erststimmeneffekt. Aber nicht nur das Alter macht es wahrscheinlicher, dass ein bestimmter Politiker bekannter wird und daher höhere Wahlchancen besitzt (während gleichzeitig die Wahrscheinlichkeit eines Titelerwerbs natürlich auch über die Zeit zunimmt), sondern auch, wenn er bereits Mitglied des Bundestags ist. ${ }^{6}$ Wir kontrollieren daher im Fall der Listenposition zusätzlich eine vorherige Mitgliedschaft im Bundestag - und finden einen sehr starken, jeglichen Titeleffekt deutlich dominierenden Effekt. Für die abhängige Variable Erststimmenergebnis konnten wir wegen Kollinearität nicht für eine vorherige Bundestagsmitgliedschaft kontrollieren.

Tabelle 4: Kausaleffekt des Erwerbs des akademischen Titels (Fixed-Effects-Model)

\begin{tabular}{|l|c|c|c|}
\hline & Relativer Listenplatz & Relativer Listenplatz & Erststimmenergebnis \\
\hline Doktortitel & $-0,027$ & $-0,004$ & 0,226 \\
\hline & $(0,448)$ & $(0,036)$ & $(0,876)$ \\
\hline Alter & $-0,040$ & $-0,036$ & 1,509 \\
\hline & $(0,001)^{* * *}$ & $(0,012)^{* * *}$ & $(0,054)^{*}$ \\
\hline Alter $^{2}$ & 0,0003 & 0,0003 & $-0,015$ \\
\hline & $(0,010)^{* * *}$ & $(0,0001)^{* * *}$ & $(0,047)^{* *}$ \\
\hline Einzug in den Bundestag & & $-0,174$ & \\
\hline & & $(0,034)^{* * *}$ & 11,109 \\
\hline Konstante & 1,421 & 1,397 & $(0,557)$ \\
\hline & $(0,000)^{* * *}$ & $(0,243)^{* * *}$ & $99(37$ Individuen $)$ \\
\hline Beobachtungen & $439(156$ Individuen $)$ & $439(156$ Individuen $)$ & \\
\hline
\end{tabular}

Anmerkungen wie Tabelle 1.

Quelle: Eigene Darstellung.

5 Aus offensichtlichen Gründen ist eine analoge Betrachtung für Wahlbewerber mit Adelstitel nicht möglich.

6 Die Literatur bestätigt einen Amtsinhaberbonus auch für den deutschen Fall (Bawn 1999; Boll/ Römmele 1994; Hainmüller/Kern/Bechtel 2006). 


\section{Fazit}

Die hier unternommene Untersuchung konnte nicht nur einen Erststimmeneffekt des Doktortitels (Schneider/Tepe 2011) auf einer sehr viel breiteren Datengrundlage bestätigen, sondern zusätzlich einen zweiten Effekt feststellen, der über die bessere Listenpositionierung von Wahlbewerbern mit Doktor- oder Professorentitel wirkt. Akademische Titelträger erringen nicht nur einen höheren Erststimmenanteil (in unserem Datensatz im Vergleich zu den anderen gewählten Wahlkreisbewerbern), sondern sie erhalten auch tendenziell bessere Listenplätze. Dies ist insofern von Bedeutung, da der Listeneffekt für alle Parteien relevant ist, während der Erststimmeneffekt fast ausschließlich für die Wahlbewerber von CDU/CSU und SPD eine Rolle spielen kann. Insgesamt zeigen sich damit auch bessere Chancen, in den Bundestag gewählt zu werden, wenn man einen akademischen Titel vorweisen kann. Unsere Untersuchung ergänzte die Betrachtung einerseits um die Listeneffekte des akademischen Titels und nahm zusätzlich auch Adelstitel in den Blick - ebenfalls mit ihren Effekten auf Erststimmenanteile und Listenpositionierungen. Hier konnten wir zwar prinzipiell gleichgerichtete Effekte auf Erststimmen, Listenrang und generell die Wahlchance konstatieren, die Effektstärke fiel jedoch insgesamt schwächer aus und blieb häufiger unterhalb konventioneller Standards statistischer Signifikanz.

Zum Abschluss unserer Analyse muss allerdings betont werden, dass damit keine Aussage darüber getroffen ist und werden kann, ob der Titel selbst ausschlaggebend für diese höhere Wahlwahrscheinlichkeit ist oder ob er nur Kandidatenmerkmale indiziert (etwa stärker ausgeprägter Ehrgeiz oder schnellere Auffassungsgabe), die dem politischen Wettbewerbsvorteil der Titelträger eigentlich kausal zugrunde liegen. So finden wir keine harte Evidenz dafür, dass es direkt der Titel ist, der eine politische Aufwärtsmobilität begründet. Die Betrugsfälle der jüngsten Vergangenheit haben jedoch überdeutlich gezeigt, dass ein unrechtmäßig erworbener Titel für eine ganz erhebliche politische Abwärtsmobilität der Betroffenen sorgen kann. Als Fazit ließe sich also festhalten: „Eventuell ist der Doktorhut gar nicht so viel wert, wie mitunter betrogen wird, um ihn zu erlangen“(Wagner 2011). 


\section{Literatur}

Bawn, Kathleen, 1999: Voter Responses to Electoral Complexity. Ticket Splitting, Rational Voters and Representation in the Federal Republic of Germany, British Journal of Political Science 29, 487-505.

Best, Heinrich/Cotta, Maurizio, 2000 a: Elite Transformation and Modes of Representation since the Mid-Nineteenth Century. Some Theoretical Considerations, in: dies. (Hrsg.), Parliamentary Representatives in Europe, 1848-2000. Legislative Recruitment and Careers in Eleven European Countries, Oxford, 1-28.

Best, Heinrich/Cotta, Maurizio, 2000 b: Parliamentary Representatives from Early Democratization to the Age of Consolidated Democracy. National Variations and International Convergence in a Long-term Perspective, in: dies. (Hrsg.), Democratic Representation in Europe, Oxford, 1-26.

Boll, Bernhard/Römmele, Andrea, 1994: Strukturelle Vorteile der Amtsinhaber? Wahlchancen von Parlamentariern im internationalen Vergleich, Zeitschrift für Parlamentsfragen 25 (4), 543-556.

Brüderl, Josef, 2010: Kausalanalyse mit Paneldaten, in: Christof Wolf/Henning Best (Hrsg.), Handbuch der sozialwissenschaftlichen Datenanalyse, Wiesbaden, 963-994.

Cotta, Maurizio/Best, Heinrich, 2000: Between Professionalization and Democratization. A Synoptic View on the Making of the European Representative, in: Heirich Best/Maurizio Cotta (Hrsg.), Parliamentary Representatives in Europe 1848-2000. Legislative Recruitment and Careers in Eleven European Countries, Oxford, 493-526.

Faas, Thorsten/Schoen, Harald, 2006: The importance of being first. Effects of candidates' list positions in the 2003 Bavarian state election, Electoral Studies 25 (1), 91-102.

Hainmüller, Jens/Kern, Holger Lutz/Bechtel, Michael, 2006: Wahlkreisarbeit zahlt sich doppelt aus - Zur Wirkung des Amtsinhaberstatus einer Partei auf ihren Zweitstimmenanteil bei den Bundestagswahlen 1949 bis 1998, in: Thomas Bräuninger/Joachim Behnke (Hrsg.), Jahrbuch für Handlungs- und Entscheidungstheorie, Wiesbaden, 11-46.

Kelley, Jonathan/McAllister, Ian, 1984: Ballot Paper Cues and the Vote in Australia and Britain. Alphabetic Voting, Sex, and Title., Public Opinion Quarterly 48, 452-466.

Mackenrodt, Christian, 2008: Wie wichtig ist die Person? Zur Bedeutung von Persönlichkeitsfaktoren von Wahlkreisbewerbern bei Bundestagswahlen, Zeitschrift für Parlamentsfragen 39 (1), 69-83. 
Manow, Philip/Flemming, Peter, 2011: Kandidaten- und Abgeordnetendatensatz Bundestag, 1949-2009, Universität Bremen/Universität Mannheim.

McDermott, Monika L., 1997: Voting Cues in Low Information Elections. Candidate Gender as a Social Information Variable in contemporary United States Elections, American Journal of Political Science 41, 270-283.

McDermott, Monika L., 2005, Candidate Occupation and Voter Information Shortcuts, Journal of Politics 67, 201-219.

Rosar, Ulrich, 2009: Fabulous Front Runners. Eine Analyse zur Bedeutung der physischen Attraktivität von Spitzenkandidaten für den Wahlerfolg ihrer Parteien und zu den Möglichkeiten zur Gegensteuerung durch das Wahlrecht, Politische Vierteljahresschrift 50, 754-773.

Schneider, Sebastian/Tepe, Markus, 2011: Dr. Right and Dr. Wrong. Zum Einfluss des Doktortitels auf den Wahlerfolg von Direktkandidaten bei der Bundestagswahl 2009, Politische Vierteljahresschrift 52, 248-285.

Wagner, Gerald, 2011: Der kann es, der ist promoviert, Frankfurter Allgemeine Zeitung, 2.11.2011, N5.

Korrespondenzanschrift:

Philip Manow

Zentrum für Sozialpolitik

Universität Bremen

Unicom-Gebäude

Mary-Somerville-Straße 5

28359 Bremen

E-Mail:manow@zes.uni-bremen.de

Peter Flemming

BITMARCK Service GmbH

Lindenallee $6-8$

45127 Essen

E-Mail: peter.flemming2@nexgo.de 